\title{
A Hadoop-Galaxy adapter for user-friendly and scalable data-intensive bioinformatics in Galaxy
}

\author{
Luca Pireddu $^{*}$ \\ CRS4, Pula, Italy \\ University of Cagliari, Italy \\ luca.pireddu@crs4.it \\ Nicola Soranzo \\ CRS4, Pula, Italy \\ nicola.soranzo@crs4.it
}

\author{
Simone Leo \\ CRS4, Pula, Italy \\ University of Cagliari, Italy \\ simone.leo@crs4.it \\ Gianluigi Zanetti \\ CRS4, Pula, Italy \\ gianluigi.zanetti@crs4.it
}

\begin{abstract}
In this work we present a strategy to integrate Hadoop-based applications into the Galaxy platform along with an extensible implementation of this adapter and related utilities. The strategy is based on the idea of introducing a new Galaxy datatype that provides a layer of indirection, thus relaxing the requirement to place data on a Galaxy-accessible file system and instead allowing the referenced data to be placed on any addressable space, including the Hadoop Distributed File System or Amazon S3. The adapter supports using Hadoop-based applications as part of Galaxy workflows. We demonstrate a practical application where this Hadoop-Galaxy adapter was used at CRS4 to accelerate the bioinformatics analysis of viral vector integration sites through the introduction of Hadoop-based computation components, while keeping the workflow under control of biologists with little specific technical training.
\end{abstract}

\section{Categories and Subject Descriptors}

J.3 [Life and Medical Sciences]: biology and genetics; D.1.3 [Programming Techniques]: concurrent programming-distributed programming

\section{General Terms}

Performance

\section{Keywords}

Bioinformatics, NGS, Galaxy, Hadoop

\footnotetext{
${ }^{*}$ Corresponding author

Permission to make digital or hard copies of all or part of this work for personal or classroom use is granted without fee provided that copies are not made or distributed for profit or commercial advantage and that copies bear this notice and the full citation on the first page. Copyrights for components of this work owned by others than the author(s) must be honored. Abstracting with credit is permitted. To copy otherwise, or republish, to post on servers or to redistribute to lists, requires prior specific permission and/or a fee. Request permissions from permissions@acm.org. $B C B^{\prime} 14$, September 20-23, 2014, Newport Beach, CA, USA.

Copyright is held by the owner/author(s). Publication rights licensed to ACM ACM 978-1-4503-2894-4/14/09 ...\$15.00.

http://dx.doi.org/10.1145/2649387.2649429.
}

\section{INTRODUCTION}

Rapid progress in biological and biomedical data acquisition technologies is turning modern biology into a dataintensive science $[11,20]$. This can be mostly attributed to the increasing diffusion of massively parallel data acquisition systems, next-generation sequencing (NGS) machines [14] being among the most cited examples. The introduction of NGS has allowed researchers to explore important, but previously inaccessible, biological questions and has paved the way to a host of significant new techniques and protocols that have a wide range of important applications in biology and medicine [19]. However, to use NGS data one has to surmount two main, interrelated, difficulties: processing complexity and dataset size. In fact, the extraction of biologically significant information from the raw sequencing data requires complex, multi-step, computationally intensive processing workflows. In its bare form, this processing is not trivial and requires specialized computing skills, such as familiarity with shell programming and high-performance computing (HPC), and knowledge about the layout of the local storage infrastructure. These issues are typically not of high interest to biologists, since they are system-level obstacles that only need to be defeated in order to achieve the final objectives of their work.

The desire to simplify such analyses from the user's point of view has historically motivated the creation of a number of graphical bioinformatics platforms - some examples are Galaxy [7], Taverna [13] and Chipster [9] - which allow the system-level details tied to implementing such processing workflows to be hidden below a high-level graphical interface. In this manner, the biologists can express the workflow they would like to run by graphically connecting operations. Galaxy in particular has been garnering a growing level of popularity among biologists in recent years, as can been seen by the growing number of related papers and of public installations (more than 50 at the time of this writing, see https://wiki.galaxyproject. org/PublicGalaxyServers for an updated list). Indeed, we have also adopted Galaxy at CRS4 for our DNA and RNA sequence processing operations.

This high-level graphical user interface isolates the users from the technical details required to execute the computation. For instance, it allows system administrators to configure the system to transparently use computational resources and methods appropriate for the tasks at hand. 
More specifically, such installations can be configured to run jobs through standard HPC batch-queue systems, thus accessing available computing cluster nodes transparently, where they may also have access to high-performance or particularly large parallel shared storage systems.

For many types of biology-related computation this arrangement is sufficient. However, NGS poses particularly challenging data throughput requirements. Consider that a modern sequencing machine can generate about $8 \mathrm{~TB}$ of raw data per week which needs to be processed and stored, so even medium-size sequencing operations can face significant operational and infrastructural challenges. The need for scalable computing solutions in this sector has prompted work in applying "BigData" technologies, especially Hadoop [8, 16], to sequencing-related operations $[12,15,17,18]$. These new Hadoop-based tools are designed to be scalable both in the amount of data to be processed and in the use of available computing resources, meaning that in general one can simply add more computing nodes to achieve higher throughput. Unfortunately, these new Hadoop-based tools are not directly compatible with Galaxy and thus cannot be natively used through its user-friendly interface for reasons discussed in more detail in Section 3.1.

In this work, we address the incompatibilities between these two systems to provide a functional and extensible integration layer, allowing users to mix and match Hadoopbased and conventional tools in their Galaxy workflows. The solution is simple to use, but requires the workflow designer to keep in mind when the data is being processed in the "conventional space" and when in the "Hadoop space" something which is done through explicit operations that are inserted in the workflow. This Hadoop-Galaxy adapter has been released as open source software (available at https: //github.com/crs4/hadoop-galaxy/ and is already available in the Galaxy Tool Shed (http://toolshed.g2.bx. psu.edu/view/crs4/hadoop_galaxy).

The rest of this article is structured as follows. Section 2 provides some background information regarding Galaxy and Hadoop. Section 3.1 describes the incompatibilities between these two systems and thus explains why this Hadoop-Galaxy adaptor is required; the rest of Section 3 describes how the adaptor works and how to use it. Section 4 then explains how to use our Hadoop-Galaxy adaptor to integrate new Hadoop-based tools with any Galaxy installation, while Section 5 follows with the details of a concrete use case we have developed at CRS4. The paper then describes related work in Section 6 and finally concludes.

\section{BACKGROUND}

\subsection{Galaxy}

Galaxy is a user-friendly bioinformatics workflow management system. Through its web interface it allows users to graphically describe workflows by drawing pipes to connect tools. Once a workflow is launched, the system can then automatically manage its execution, running steps in an appropriate order and, when dependencies allow, executing them concurrently. The platform is extensible, allowing users to integrate new tools which can then be included in new workflows. In fact, there exists a large communitymanaged library of tools that are already integrated and thus are ready to use or easily added after installation.
To support managing workflows in this automated and extensible manner, the platform imposes a model on the tools that are integrated and the datasets that they process and generate. Specifically, tools are called by command line, where Galaxy provides them with input and output dataset paths and user-selected options. In the Galaxy model, tools that are invoked in succession in a workflow "communicate" - as in passing datasets from one to the next - by sharing file system space. So, Galaxy is configured to use a directory as a workspace, where it creates paths for the various datasets that are generated during its operation. It is important to note that Galaxy needs to be able to access and read these dataset files and thus the workspace needs to be on a file system mounted on the system where the platform is running.

\subsection{Hadoop}

Hadoop is a framework and a platform for large-scale data processing. Although with its rising popularity the Hadoop ecosystem has been growing with numerous tools and variations, the crux of the system - and the only parts relevant to this work - are its open source implementation of MapReduce [6] and the Hadoop Distributed File System (HDFS).

The MapReduce implementation provides a framework into which an application can install appropriate map and reduce functions that in combination compute a desired result. These functions must be implemented without sideeffects, so that the framework can distribute their execution automatically to any available node and execute multiple instances concurrently by splitting the input into blocks; it can even re-execute the functions on the same input data for instance, in the case of stragglers or to recuperate dead tasks - by adopting appropriate measures to ensure idempotency and safe concurrency. Among these measures we find that each work task executed by the framework writes to its own output file. As such, when processing data with Hadoop MapReduce datasets are typically kept in directories, which may contain any number of files, each of which contains a part of the entire dataset.

To maximise its effectiveness, Hadoop MapReduce is complemented by the HDFS. This file system distributes its data in blocks over the entire Hadoop cluster. This storage strategy creates opportunity for executing the map and reduce functions directly on the node containing the data block to be processed, thus reducing the network I/O generated by the application. In addition, by its architecture HDFS makes its total bandwidth scale with the number of nodes in the cluster. These features make it the standard file system used for Hadoop clusters.

\section{GALAXY-HADOOP INTEGRATION}

\subsection{Incompatibilities}

The execution models used by Galaxy and Hadoop are incompatible, meaning that even Hadoop-based tools that are invoked from the command-line cannot be easily integrated into a Galaxy workflow. There are two main issues that keep this integration from working, both pertaining to the two platforms' view of datasets. The first is that the two systems store datasets differently. Specifically, Galaxy assumes that a dataset is entirely contained in a single file, while Hadoop MapReduce purposefully splits datasets into multiple files - possibly bundled into a single directory - to 
be more amenable to processing in multiple parallel tasks. Because of this incompatibility, one cannot present the output of a Hadoop job to Galaxy as a single dataset. Though at the time of this writing work is being done by the Galaxy developers to resolve this limitation, in the way of multi-file datasets, it is still in the early stages of development (see Pull Request \#386 in the galaxy-central repository for a complete description: http://goo.gl/UVuRq $7^{1}$ ). The second issue relates to the file system where the data is stored and the fact that Galaxy expects to be able to access it directly. This issue is a problem since Galaxy can only access conventional file systems that are mounted on the machine where the server runs. On the other hand, Hadoop clusters are typically configured to use the HDFS for storage, which cannot be readily accessed by Galaxy. In fact, HDFS was not designed to be mounted on a system and is instead accessed through its own client application programming interface (API) or web service. Although some methods exist to mount HDFS like a conventional file system - e.g., NFS gateway to HDFS or mounting HDFS through Linux Filesystem in Userspace (FUSE) - using them would entail putting the entire Galaxy workspace on HDFS and these solutions would not be suitable to handle such file system activity. A similar argument can be made for Amazon S3 storage, which is also accessible transparently to Hadoop programs but not Galaxy.

\subsection{Solution by Indirection: Pathsets}

Due to the incompatibilities between Hadoop's and Galaxy's tool models, Galaxy cannot use Hadoop-based tools directly. However, as David Wheeler once said, "All problems in computer science can be solved by another level of indirection" [21]. We followed this advice and introduced the pathset data type. In brief, a pathset is a list of one or more complete paths that define a single dataset. Concretely, it is an ordered list of uniform resource identifiers (URIs), each identifying one or more files or directories, augmented by the ability to use the shell-like wildcards. The dataset defined by the pathset can be materialized by concatenating the contents of all the files spanned by the pathset: therefore, the order in which the URIs are listed is important. When a URI references a directory the files in the entire directory hierarchy are included in the pathset as if by recursive traversal. When selecting paths through patterns or recursion, the elements are included in alphabetical order. In addition to the paths themselves, the pathset includes some minimal metadata, such as the format of the data being referenced.

The pathset is implemented as a Python class which, in addition to basic operations such as adding, removing and iterating over URIs, implements a text-based serialization. An example of the serialization format is shown in Fig. 1). Such a file can be readily handled as a dataset by Galaxy, for which we have defined a pathset data type to ensure that only pathset-compatible tools can be connected to each other.

By executing Hadoop-based tools in Galaxy through an adapter, we are able to use the pathsets - which are singlefile entities - to reference the real data which may be located

\footnotetext{
${ }^{1}$ https://bitbucket.org/galaxy/

galaxy-central/pull-request/386/

dataset-collections-initial-models-api/
}

on file systems that can be referenced through a URI and split between any number of files and directories.

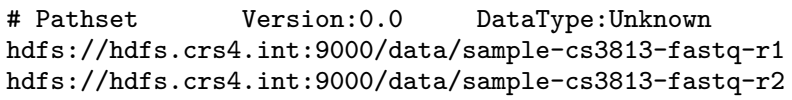

Figure 1: Example of a pathset file. The header begins with a sequence to identify the file type, the version of the pathset format, and then contains metadata describing the format of the referenced data.

\subsection{Hadoop-Galaxy Adapter}

Although the pathset datatype gives us a way to indirectly reference data that is too large to be easily placed in Galaxy's workspace or that is located on file systems that cannot be mounted, on its own it is insufficient to allow Galaxy to use Hadoop-based tools in its workflows. To complete the solution, we have implemented an adapter program - the Hadoop-Galaxy adapter - that can "dereference" the input and output pathsets and pass the contents to the Hadoop-based program that needs to run.

The Hadoop-Galaxy adapter can work as an executable or as a Python function called by another program. To use it, one specifies: the input pathset, referencing the input data; the output pathset, which will reference the output data after execution; a location where the Hadoop program can write its output data, typically on HDFS; finally, the name or path of the executable of the Hadoop-based tool and any additional arguments it needs to operate. The adapter will stage the data output directories as needed and write their path to the output pathset. It will then read the input paths from the input pathset and then call the executable, passing the real data input and output paths as arguments.

A configuration file can be provided either by commandline argument or, more conveniently for Galaxy-related applications, by setting an environment variable which could be used to simultaneously affect all Hadoop-based tools integrated through our adapter. Through this configuration file the user can vary several operational aspects, including which Hadoop cluster to use and the environment variables to set when the Hadoop-based tools are run. The job's output data path is decided autonomously by the adapter based on the configuration provided by the user. This setting is quite important when operating at scale because our integration technique, at the moment, does not provide garbage collection for the data referenced by the pathsets. Indeed, a notable shortcoming of our system in its current form is that when a pathset dataset is deleted by Galaxy, the data it referenced remains in existence and occupies disk space. To alleviate this situation, the user can configure a directory where all Hadoop-based tools write, knowing they can schedule occasional clean-up sessions to delete old intermediate data. This strategy is being successfully used at CRS4 for its production sequencing pipeline, which uses Galaxy and Hadoop for many of its processing stages.

\subsection{Utility Programs}

In addition to the adapter itself, Hadoop-Galaxy provides a number of utility tools to facilitate the integration of Hadoop-based and regular tools in the same workflow. In the following paragraphs we list and briefly describe them. 
make_pathset: a tool to create a new pathset that references files or directories provided as input. The input can be provided by connecting the output of another Galaxy tool, thus providing a bridge to take a "regular" Galaxy dataset and pass it to a Hadoop-based tool. Alternatively, the input can be given as a direct parameter, which can be useful, for instance, for creating workflows where the user specifies the input path as an argument or when performing ad hoc analysis with Galaxy.

cat_paths: a tool to take the list of part files that make up a dataset and concatenate them into a single file. This tool effectively provides the operation inverse to make_pathset: while make_pathset creates a level of indirection by writing a new pathset that references data files, cat_paths copies the referenced data into a single file. The new destination file exists within Galaxy's workspace and can therefore be used by other standard Galaxy tools. Thus, with the combination of make_pathset and cat_paths, a single Galaxy workflow can mix processing with standard tools and Hadoop-based operations.

However, since Hadoop-based processing can generate a lot of data, copying it to the Galaxy workspace one chunk at a time in a serial process can be very time-consuming. To ameliorate this issue, cat_paths provides a distributed mode that uses the entire Hadoop cluster to copy data chunks to the same file in parallel. For this feature to work, the Galaxy workspace must be on a parallel shared file system accessible by Hadoop nodes. When applicable, its effects can be significant: with datasets on CRS4's computing cluster we have observed speed-ups of up to 40x.

put_dataset: a tool to copy data from the Galaxy workspace to Hadoop storage. It is possible that in some configurations the Galaxy workspace may be stored on a volume that is directly accessible by the Hadoop cluster. In this case, before feeding the data as input to a Hadoop-based program it is necessary to copy it from Galaxy's storage to the Hadoopaccessible storage. For this task Hadoop-Galaxy provides the put_dataset tool, which works in a way analogous to the command

hadoop dfs -put <local file> <remote file>

The put_dataset operation receives a pathset as input and creates a new one as output, where for each input path element there is a corresponding output path for the file at its new location. It is important to keep in mind that this copy operation is serial in nature, as it needs to run directly on the server that has access to the Galaxy workspace. As such, it provides limited bandwidth and can be time-consuming for large files. If large files need to be passed between the Galaxy and Hadoop workspaces, it is a much faster solution to have the Galaxy workspace on a parallel shared file system that can be accessed directly by the Hadoop cluster, thus eliminating the need for put_dataset.

split_pathset: a tool that can be used in a Galaxy workflow to split a pathset into two parts based on path or filename. It allows the user to define a regular expression as a test: the path elements are then accordingly placed in the "match" or "mismatch" output pathset. This type of tool is handy in cases when the Hadoop tool associates a meaning to the output file. For instance, some tools in the Seal suite for processing sequencing data [15] can separate DNA fragments based on whether they were produced in the first or second sequencing phase (i.e., read 1 or read 2) putting them each under a separate directory. This tool allows a workflow to split the Galaxy dataset into two sets according to this criteria.

dist_text_zipper: a tool for parallel (Hadoop-based) compression of text files. Although this tool is not required for the use of Hadoop-Galaxy in user workflows, it is a generally useful utility that doubles as an example illustrating how to integrate Hadoop-based tools with Galaxy using our adapter.

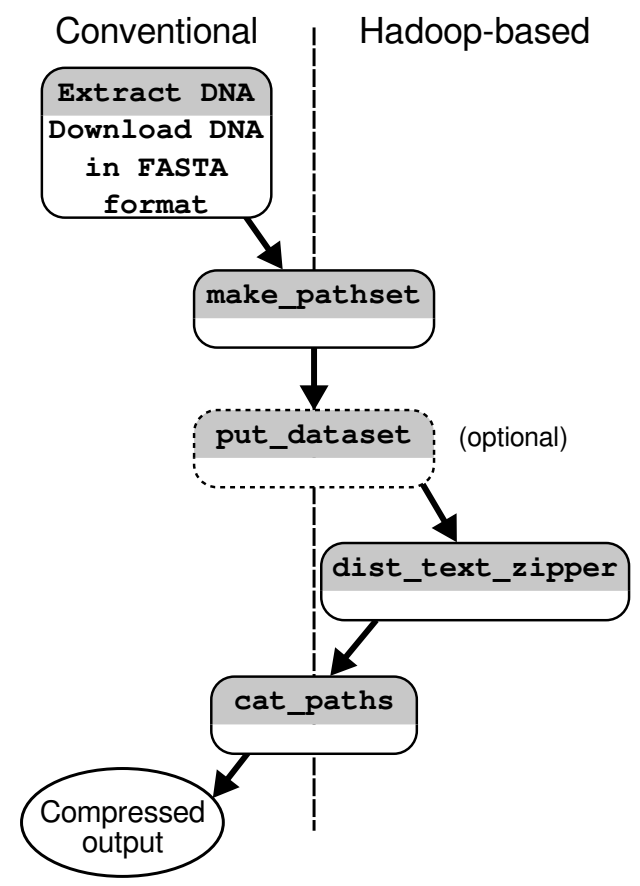

Figure 2: A Galaxy workflow that fetches a dataset (DNA sequences) in text format, using a standard Galaxy tool (Fetch Sequences $\rightarrow$ Extract Genomic $D N A$ ), and uses Hadoop-Galaxy to compress them using a Hadoop cluster. The dist_text_zipper program (run over our Hadoop-Galaxy adapter) is used to compress the data, while the make_pathset and cat_paths utilities are used to provide the appropriate data bridge between the Galaxy workspace and the Hadoop file system. The optional put_dataset component is only needed if the Hadoop cluster cannot access the Galaxy workspace directly.

\section{INTEGRATING NEW TOOLS}

The components of the Hadoop-Galaxy project are designed to simplify the integration of Hadoop-based tools with Galaxy. The dist_text_zipper utility, distributed with Hadoop-Galaxy, provides a clear example. The Hadoopbased program has a regular command-line interface, receiving input path(s) and an output path - both of which must be accessible by the Hadoop nodes and may be on an unconventional file system such as HDFS. To use the program through Galaxy, it is sufficient to write a normal tool definition in XML; but, rather than invoking the program directly, it should be called through the hadoop_galaxy adapter (illustrated in Fig. 3). No modifications to the program are necessary. Also, passing additional arguments to the tool 


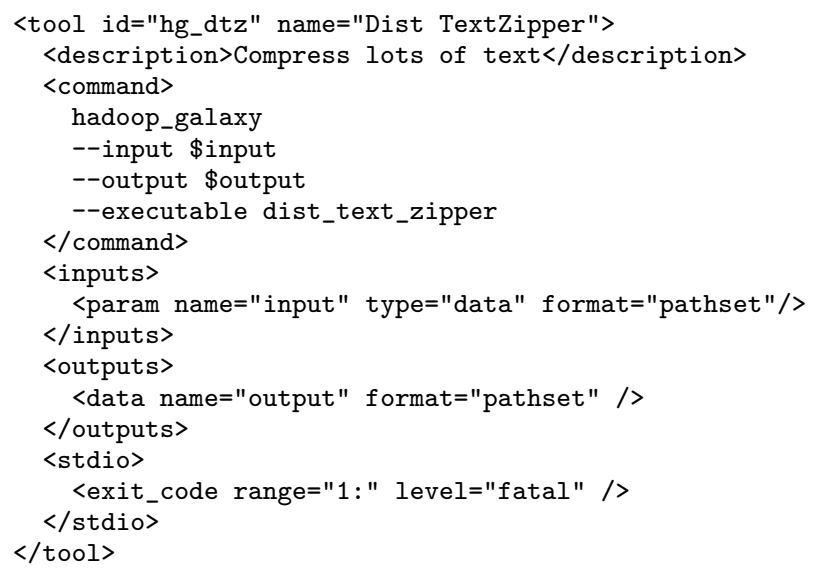

Figure 3: Example of a Hadoop-based tool (dist_text_zipper) integrated as a Galaxy tool. Notice how the tool is called through the hadoop_galaxy adapter, rather than being invoked directly.

is simple since the adapter forwards any arguments that it does not recognize. In its standard form, the adapter assumes that the slave program can be called in the form:

cmd_name [options] input output

To accommodate programs with a different interface (for instance, a common case may be programs that identify the desired output path with a -o option) the Hadoop-Galaxy adapter supports user-provided pluggable command runners that can implement the required interface, though they need to be written in Python.

For additional examples showing how to use Hadoop-Galaxy to integrate Hadoop-based tools with Galaxy, developers can look at the adaptors for the Seal suite of tools for processing DNA sequencing data (https://github.com/crs4/ seal-galaxy). These tools and wrappers are used to process the data produced by CRS4's three Illumina HiSeq 2000 high-throughput sequencers.

\section{SAMPLE USE CASE}

At CRS4, we recently developed a custom bioinformatics workflow for the analysis of viral vector integration sites (ISs) to assess the safety of a novel hematopoietic stem cell gene therapy (HSC-GT) treatment for metachromatic leukodystrophy (MLD) [2]. The patient is infused with HSCs modified by viral vectors, enabling them to express a particular enzyme whose absence leads to the condition. Despite its efficacy in treating the disease, however, GT can cause serious side effects: changes in the genomic area close to the vector's IS can trigger the expression of harmful genes, a phenomenon known as insertional mutagenesis. Thus, the ability to predict and monitor the genomic distribution of viral vectors is crucial to the safety of the procedure. IS identification starts in the wet lab, where host DNA is amplified via polymerase chain reaction (PCR) and then sequenced, which produces a considerable amount of plain text data (sequencing reads). The rest of the analysis is done in silico: bioinformatics tools are needed to remove viral and artificial fragments from the reads, align them to a reference genome, apply a series of filters to ensure unambiguous map-

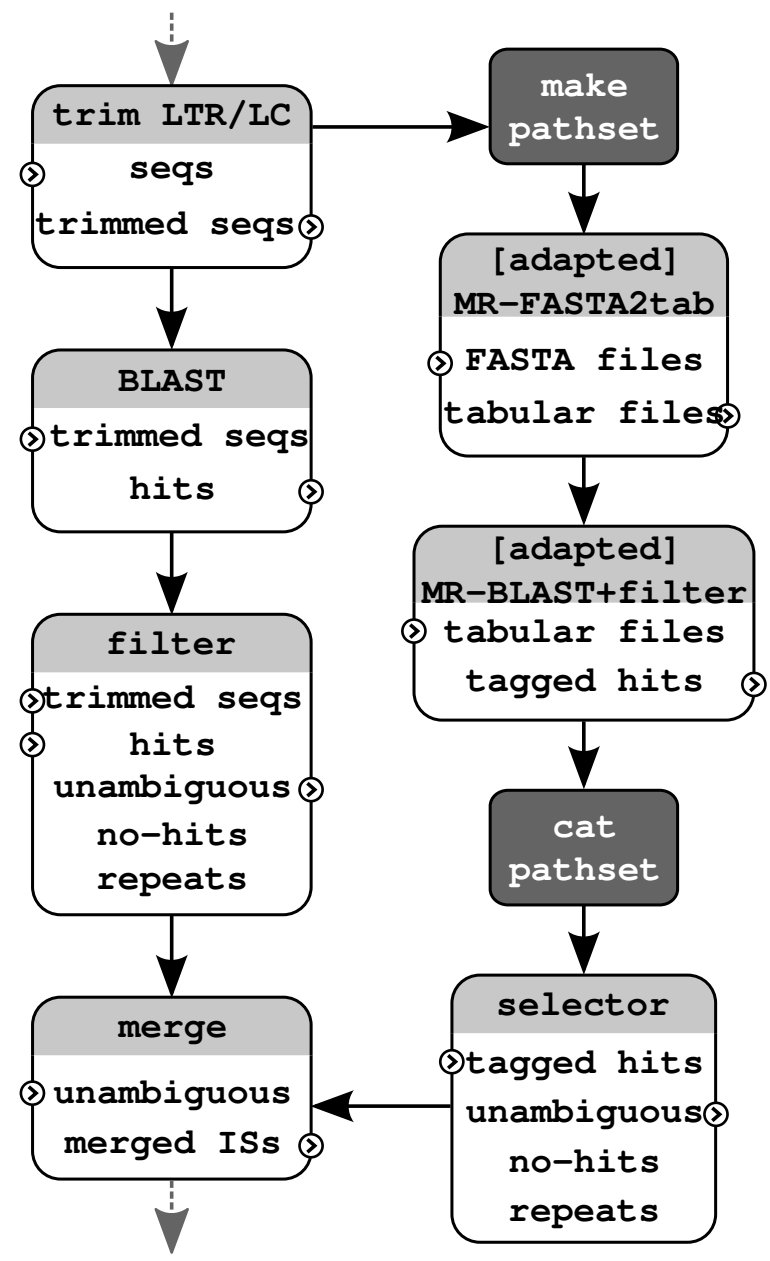

Figure 4: The BLAST and filter section of VISPA's Galaxy workflow, implemented with ordinary singlecore tools (left flow) and with MapReduce applications (right flow). Hadoop-Galaxy adapters and tools allow to integrate the latter into the Galaxy workflow unchanged. 


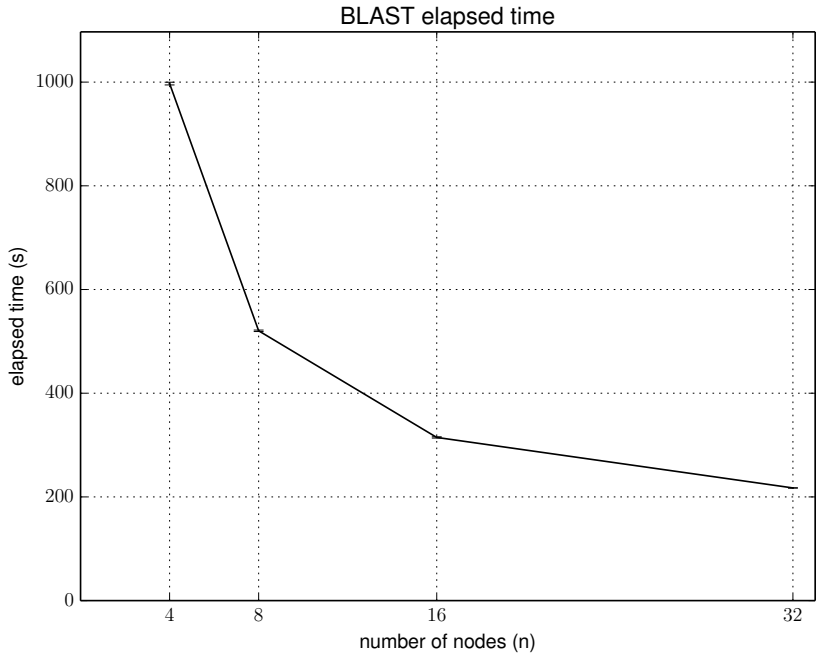

Figure 5: Time required to align and filter 2048 64base sequences, for different cluster sizes, with our distributed version of BLAST (average over three repeated runs; used BLAST version 2.2.21). Four CPU cores were used on each node. The suboptimal speedup is related to implementation details of the MapReduce application and is not tied to the use of the Hadoop-Galaxy adapter.

ping, merge equivalent ISs and annotate them with nearby genomic features (e.g., genes). Our pipeline, called VISPA (https://github.com/crs4/vispa) takes care of the computational part of the analysis, from raw sequencing data to annotated ISs.

While most of the analysis steps could be implemented efficiently as ordinary single-core programs, the alignment and filtering step proved to be significantly more challenging. During the experiments carried out in the course of the MLD study, nearly 14 million sequences had to be aligned to the human reference genome with BLAST [1]: on a single processor, such a task can take more than a year to complete, an amount of time which is incompatible with the turnaround requirements of clinical trials. Although BLAST can optionally use multiple CPUs, in practice this leads to minor performance increases, since only a small part of the code is multi-threaded. Thus, to achieve significant speedups, the set of input sequences needs to be split into multiple files, each of which must be fed to a separate BLAST process. Since Hadoop already provides this functionality in a manner suitable for scaling up to a large number of CPU cores distributed across different machines, we reimplemented the alignment and filtering steps on this framework. For consistency with the rest of the pipeline, we used Pydoop [10], a Python API for Hadoop developed at CRS4. Figure 5 compares the time required to align and filter 2048 64-base long sequences to the human genome (hg19 assembly) for varying Hadoop cluster sizes. Input sequences were randomly generated as sub-samples of chromosome 22, with a mismatch rate of one base every two sequences. As shown in the figure, the running time can be reduced by adding more nodes to the cluster: in this case, due to the small size of the test dataset, saturation is approached rather quickly as the overhead introduced by the framework becomes comparable with the core computation; in real-world applications, substantial gains can be obtained up to much larger cluster sizes.

Despite the obvious advantages of the distributed implementation, its execution model does not allow its immediate integration into the main Galaxy workflow: Hadoop applications read one or more input files from HDFS and write one output file per task on an HDFS directory; Galaxy, on the other hand, normally operates on datasets that reference files uploaded to its local file system. Figure 4 depicts how the Hadoop-Galaxy adapter allowed us to integrate the tools without modifying the MapReduce code.

The left flow corresponds to a "standard" Galaxy implementation: trimmed sequences output by the previous step are passed to the BLAST tool [5], while a subsequent filtering step classifies them as unambiguously aligned, not mapped or repeats (i.e., with multiple high-score mappings) and writes them to three separate files. Unambiguously aligned sequences are then passed to the IS merging step, while the other two sets are kept for statistical purposes. Note that the filtering step needs the trimmed sequences to compute their length.

The right flow shows the MapReduce implementation of the BLAST and filtering steps, as well as their integration into Galaxy. Since a one-record-per-line format greatly simplifies Hadoop I/O, it is useful to have a separate normalization step do the conversion from FASTA to a tabular format which can subsequently be used by multiple sequence processing application; on the other hand, since the filtering step performs the same operations on the hits corresponding to each sequence, it is more efficient to merge it with the alignment step, so that it can take advantage of the already distributed workload. Since Hadoop writes a separate output file for each concurrent task, classification is done by adding a tag to each hit, so that it can easily be dispatched to the correct output file by a subsequent selector step; due to its relatively light workload, the latter does not need to be parallelized.

The first step to integrate the MapReduce tools into Galaxy consists of adding a make_pathset tool after the trimmer, which builds a pathset containing the path of the trimmed sequences file; the fasta-to-tabular converter and the alignment and filtering tools are wrapped via the hadoop_galaxy tool (see Fig. 3), so that they can operate on pathsets; finally, cat_paths concatenates all files output by the alignment and filtering step, producing an ordinary dataset that can be passed as input to the selector.

\section{RELATED WORK}

The integration of Hadoop computational back-ends in Galaxy is a recurring theme in the Galaxy mailing lists. However, at the best of our knowledge, the only published work on the subject is a paper of Chen et al. [4] on a cloud-based image processing toolbox obtained by integrating Galaxy, Hadoop and their proprietary image processing tools. Their motivation was to provide users with a toolbox that would simplify the design and execution of complex image processing tasks using scalable cloud computation capacity. The solution proposed, based on the wrapping of their image processing programs as galaxy programs, is, however, very specialized to their application and does not 
consider the general problem of integrating Hadoop-based tools in standard Galaxy workflows.

\section{CONCLUSIONS AND FUTURE WORK}

We have presented a strategy and a functional package for easily integrating Hadoop-based applications within the Galaxy bioinformatics platform. The integration is based on the idea of introducing a layer of indirection which is handled through an adapter program, and it has been shown to work through the description of a concrete use case where a significant workflow speed-up was obtained without sacrificing user-friendliness.

Though the Hadoop-Galaxy integration presented in this work is already functional, there are several ways in which we plan to improve it. We intend to integrate the pathset data type with the Galaxy garbage collector, thus eliminating the problem of having to manually delete data when it is no longer useful. Other improvements will go in the direction of a tighter functional integration with Galaxy, which will require a richer implementation of the pathset datatype within Galaxy and will provide new features such as support for viewing excerpts of the data directly from within Galaxy (the user currently sees excerpts of the serialized pathset file) and tighter type checking, thus only allowing workflow connections between operations and pathsets that reference compatible data formats.

The Hadoop-Galaxy source code is available at https: //github.com/crs4/hadoop-galaxy. The software is also available through the Galaxy Tool Shed [3] (http://toolshed. g2.bx.psu .edu), so it can be easily added to a custom Galaxy installation. Once installed, the new "pathset" datatype and Hadoop-Galaxy tools will be available, thus allowing the user to build workflows integrating both regular and Hadoop-based tools.

\section{ACKNOWLEDGMENTS}

Parts of L.P.'s and S.L.'s activities were performed within the context of the Ph.D. program in Biomedical Engineering at the University of Cagliari, Italy.

\section{REFERENCES}

[1] S. F. Altschul, W. Gish, W. Miller, E. W. Myers, and D. J. Lipman. Basic local alignment search tool. J. Mol. Biol., 215(3):403-410, 1990.

[2] A. Biffi, E. Montini, L. Lorioli, M. Cesani, F. Fumagalli, T. Plati, C. Baldoli, S. Martino, A. Calabria, S. Canale, F. Benedicenti, G. Vallanti, L. Biasco, S. Leo, N. Kabbara, G. Zanetti, W. Rizzo, N. Mehta, M. Cicalese, M. Casiraghi, J. Boelens, U. Del Carro, D. Dow, M. Schmidt, A. Assanelli, V. Neduva, C. Di Serio, E. Stupka, J. Gardner, C. von Kalle, C. Bordignon, F. Ciceri, A. Rovelli, M. Roncarolo, A. Aiuti, M. Sessa, and L. Naldini. Lentiviral hematopoietic stem cell gene therapy benefits metachromatic leukodystrophy. Science, 341(6148):1233158, August 2013.

[3] D. Blankenberg, G. Von Kuster, E. Bouvier, D. Baker, E. Afgan, N. Stoler, J. Taylor, and A. Nekrutenko. Dissemination of scientific software with Galaxy ToolShed. Genome Biol., 15:403, 2014.
[4] S. Chen, T. Bednarz, P. Szul, D. Wang, Y. Arzhaeva, N. Burdett, A. Khassapov, J. Zic, S. Nepal, T. Gurevey, and J. Taylor. Galaxy + Hadoop: Toward a collaborative and scalable image processing toolbox in cloud. In A. R. Lomuscio, S. Nepal, F. Patrizi, B. Benatallah, and I. Brandić, editors, Service-Oriented Computing - ICSOC 2013 Workshops, volume 8377 of Lecture Notes in Computer Science, pages 339-351. Springer International Publishing, 2014.

[5] P. J. A. Cock, B. A. Grüning, K. Paszkiewicz, and L. Pritchard. Galaxy tools and workflows for sequence analysis with applications in molecular plant pathology. PeerJ, 1:e167, 2013.

[6] J. Dean and S. Ghemawat. Mapreduce: Simplified data processing on large clusters. Commun. ACM, 51(1):107-113, Jan. 2008.

[7] J. Goecks, A. Nekrutenko, J. Taylor, and the Galaxy Team. Galaxy: a comprehensive approach for supporting accessible, reproducible, and transparent computational research in the life sciences. Genome Biol., 11(8):R86, 2010.

[8] Hadoop. Welcome to Apache Hadoop, 2014. [Online; accessed 30-May-2014].

[9] M. A. Kallio, J. Tuimala, T. Hupponen, P. Klemela, M. Gentile, I. Scheinin, M. Koski, J. Kaki, and E. Korpelainen. Chipster: user-friendly analysis software for microarray and other high-throughput data. BMC Genomics, 12(1):507, 2011.

[10] S. Leo and G. Zanetti. Pydoop: a Python MapReduce and HDFS API for Hadoop. In Proceedings of the 19th ACM International Symposium on High Performance Distributed Computing, pages 819-825. ACM, 2010.

[11] V. Marx. Biology: The big challenges of big data. Nature, 498, June 2013.

[12] A. O'Driscoll, J. Daugelaite, and R. D. Sleator. 'Big Data', Hadoop and cloud computing in genomics. J. Biomed. Inform., 46(5):774-781, Oct. 2013.

[13] T. Oinn, M. Greenwood, M. Addis, M. N. Alpdemir, J. Ferris, K. Glover, C. Goble, A. Goderis, D. Hull, D. Marvin, P. Li, P. Lord, M. R. Pocock, M. Senger, R. Stevens, A. Wipat, and C. Wroe. Taverna: lessons in creating a workflow environment for the life sciences. Concurr. Comput., 18(10):1067-1100, 2006.

[14] C. S. Pareek, R. Smoczynski, and A. Tretyn. Sequencing technologies and genome sequencing. $J$. Appl. Genet., 52(4):413-435, Dec. 2011.

[15] L. Pireddu, S. Leo, and G. Zanetti. Seal: a distributed short read mapping and duplicate removal tool. Bioinformatics, 27(15):2159-2160, 2011.

[16] E. Sammer. Hadoop Operations. O'Reilly Media, Inc., 1st edition, 2012.

[17] S. Schonherr, L. Forer, H. Weiszensteiner, F. Kronenberg, G. Specht, and A. Kloss-Brandstatter. Cloudgene: A graphical execution platform for mapreduce programs on private and public clouds. BMC Bioinformatics, 13(1):200, 2012.

[18] A. Schumacher, L. Pireddu, M. Niemenmaa, A. Kallio, E. Korpelainen, G. Zanetti, and K. Heljanko. SeqPig: simple and scalable scripting for large sequencing data sets in Hadoop. Bioinformatics, 30(1):119-120, Jan. 2014 . 
[19] W. W. Soon, M. Hariharan, and M. P. Snyder. High-throughput sequencing for biology and medicine. Mol. Syst. Biol., 9:640, Jan. 2013.
[20] K. M. Tolle, D. S. W. Tansley, and A. J. G. Hey. The Fourth Paradigm: Data-Intensive Scientific Discovery. Proc. IEEE, 99(8):1334-1337, 2011.

[21] Wikipedia. David Wheeler (computer scientist), 2014. [Online; accessed 28-May-2014]. 